\title{
Enquête
}

Archives de la revue Enquête

4 | 1996

La ville des sciences sociales

\section{Les compétences de rassemblement}

Une ethnographie des lieux publics

Skills of gathering. An ethnography of public places

Isaac Joseph

\section{CpenEdition}

Journals

Édition électronique

URL : http://journals.openedition.org/enquete/773

DOI : $10.4000 /$ enquete. 773

ISSN : 1953-809X

Éditeur :

Cercom, Éditions Parenthèses

Édition imprimée

Date de publication : 1 novembre 1996

Pagination : 107-122

Référence électronique

Isaac Joseph, « Les compétences de rassemblement », Enquête [En ligne], 4 | 1996, mis en ligne le 11 juillet 2013, consulté le 10 décembre 2020. URL : http://journals.openedition.org/enquete/773 ; DOI : https://doi.org/10.4000/enquete.773

Ce document a été généré automatiquement le 10 décembre 2020. 


\title{
Les compétences de rassemblement
}

\author{
Une ethnographie des lieux publics \\ Skills of gathering. An ethnography of public places \\ Isaac Joseph
}

1 Un site comme la Gare du Nord est un objet urbain de plein droit. Dans la ville, éminemment, comme pôle et centralité. De la ville, jusqu'à la caricature, comme complexe d'échange ou « lieu-mouvement » selon le vocabulaire des transporteurs. Site singulier, par les traits qui l'inscrivent dans l'univers des grandes métropoles (c'est la troisième gare du monde et la première par ses flux en Europe) et par la multiplicité des démarches disciplinaires qu'implique sa description rigoureuse (celle de l'ingénieur et celle de l'architecte, celle de l'urbaniste et celle du sociologue). Site urbain dont l'observation confirme les grandes catégories d'analyse de l'urbanité selon la tradition de l'écologie urbaine depuis Robert Park et Louis Wirth: hétérogénéité des populations, avec sa conséquence, la proximité de l'étranger ; densité des relations et donc opacité relative de chacune d'elles ; superficialité des échanges qui se traduit aussi bien par leur nivellement que par leur excentricité réactive et par la force attribuée à « l'excitation formelle de la frontière, du commencement et de la fin ${ }^{1}$ ».

2 Quelles sont les conditions de possibilité d'une enquête sur un site de ce type ${ }^{2}$ ? Et si la question de l'accord doit occuper une place centrale dans les sciences sociales ${ }^{3}$, que nous dit ce site de l'accord avec tout un chacun? Accord non pas toujours avec un « autrui » puisque l'univers de la circulation nous met aussi bien en présence de "n'importe qui », c'est-à-dire de "non-personnes ». Accord non comme engagement de la volonté, mais comme résultat d'une éthologie de la coprésence entre " unités véhiculaires ${ }^{4}$ ». Quelles sont les propriétés du rassemblement et les ressources régulatrices de l'accessibilité ? Nulle part mieux que dans une gare, ne se vérifie l'idée selon laquelle un espace public est un agencement de domaines contestés ou contestables. L'accessibilité ne peut s'y réduire à la capacité d'un lieu à interagir avec d'autres lieux. Cette première définition, géographique et fonctionnelle, renvoie à la ville comme espace de circulation et de déplacements. Mais l'accessibilité est également la qualité d'intelligence partagée d'un espace de communication et désigne alors les capacités d'une prestation à ne pas être simplement un "message», d'un équipement à être plus qu'un instrument, et les 
ressources qu'ils offrent tous deux pour confirmer ou réguler les dimensions dialogiques de l'espace d'activité. À partir de la gare, il s'agit donc de comprendre comment la ville peut modifier notre perception du social et de l'histoire sociale, d'analyser la construction de l'urbanité dans ses catégories propres et de retrouver la trace de l'expérience urbaine dans les enquêtes sur les compétences sociales des citadins.

\section{Rassemblement et action conjointe}

Pour dessiner le paysage dans lequel s'exercent ces compétences, Bernard Lepetit proposait de retenir une série de traits relativement familiers à la microsociologie d'inspiration interactionniste : ajustement à une pluralité de champs normatifs et à une multiplicité de "mondes", jugement contextualisé et activité située, moments comme configurations de positions, pouvoir inégalement distribué de définir les situations ${ }^{5}$. Ces traits, qui correspondent à une vision de la vie sociale faite de situations et d'épreuves (du jugement, du sens commun et de l'action), conviennent à l'étude de ce que Goffman a appelé l'ordre de l'interaction ou l'organisation sociale des rencontres. Ils permettent de décrire rigoureusement un domaine d'activités du citadin que l'anthropologie urbaine désigne du terme de "relations de trafic", domaine privilégié d'une éthologie sociale avec ses différentes formes de coprésence ou de rassemblement ${ }^{6}$.

Un rassemblement n'est ni un groupe ni une population. C'est une configuration de positions et de mouvements dont le modèle est plutôt la file d'attente dans un espace public ou la réunion dans un espace de travail. Dans un groupe, les individus se perçoivent comme membres, perçoivent l'organisation comme une entité collective distincte, séparée des rapports particuliers qu'ils entretiennent entre eux, et attendent un soutien moral de leur identification au groupe. Ces caractéristiques peuvent se retrouver dans l'univers des rassemblements s'ils sont appelés à se reproduire, mais elles ne disent rien de leur structure propre. Le fait de se trouver ensemble peut n'être qu'une étape de la vie d'un groupe, en revanche, le fait de se quitter signe la fin d'une rencontre. De même, l'adhésion aux normes de prise de parole et de circulation de la parole n'est pas essentielle à la vie d'un groupe, pas plus que l'allocation d'une position dans l'espace où se produit le rassemblement ou la gestion des embarras qui viennent le troubler. Quelle que soit la possibilité pour le sociologue de décrire partie des événements qui se produisent en situation comme la traduction d'affiliations individuelles à des groupes différents, le domaine régi par les «normes de conjonction» ne saurait se déduire des connaissances acquises en matière de normes d'appartenance ou d'affiliation. Autrement dit, il faut distinguer les formes de l'accord qui sous-tendent une action collective, de celles que produit une action conjointe.

5 Les situations sociales susceptibles d'être décrites comme des formes d'action conjointe peuvent être des situations de travail ou de jeu, mais l'écologie des activités dont elles relèvent n'est pas sans rapport avec la définition de l'écologie urbaine proposée par l'école de Chicago. Moins que des rapports d'une population à son territoire et des formes d'adaptation qui en découlent, l'écologie urbaine entendait traiter des formes régulières d'ajustement ou de conflit produites par la coexistence de populations différentes sur un même territoire. On peut dire que la microsociologie maintient cette définition formelle mais s'intéresse à l'alignement des conduites en situation plutôt qu'à celle de communautés dans l'espace social de la ville. Changement de focale si l'on veut : il s'agit de décliner le trait (structural) de l'hétérogénéité des populations en milieu urbain pour 
comprendre les formes quotidiennes ou «mineures » de proximité avec l'étranger. Le couple conceptuel, devenu fameux depuis le travail de Jean-Claude Chamboredon et Madeleine Lemaire ${ }^{7}$, de la proximité spatiale et de la distance sociale, est assorti dans cette microécologie des interactions d'une condition commune de visibilité (d'observabilité) mutuelle qui constitue le cadre et l'« équipement » du passant dans son activité cognitive et pratique d'adaptation à un territoire lui-même conçu comme région de significations.

6 L'ethnographie des espaces publics met ainsi en scène une vision de la ville différente et complémentaire de la mosaïque de territoires élaborée à partir des monographies de quartiers. La caractéristique première des lieux publics, la norme affichée dont nous faisons l'expérience dans les encombrements et les «embarras » de la circulation, c'est qu'ils sont accessibles à quiconque. C'est là un principe d'ordre (l'égalité d'accès) et une contrainte d'usage (les initiatives non autorisées). On peut distinguer formellement, dans toute situation de coprésence en public, des participants non ratifiés, des personnes dont la présence n'est pas autorisée, des intrus ou des exclus, et des participants qui sont apparemment «à leur place ». Mais cette distinction n'est pas liée à l'appartenance à tel ou tel groupe et n'oppose pas des membres et des non-membres. Le déterminisme à l'œuvre est un déterminisme situationnel qui tient à la capacité à moduler ou "modaliser " ses engagements (distraction, évitement, indifférence civile, "réserves sûres", conversation), autrement dit à les focaliser en jouant des prises et des plis du milieu de visibilité et d'observabilité réciproque.

«Plutôt que d'opposer les interactions en face à face exceptionnelles à celles qui sont routinières, je propose de distinguer les interactions non focalisées et les interactions localisées. Les interactions non focalisées sont ces formes de communication interpersonnelle qui résultent de la simple coprésence. Par exemple deux personnes qui ne se connaissent pas et qui, d'un coin d'une pièce à l'autre observent la façon dont ils sont habillés, leurs attitudes et leur allure générale, chacun modifiant sa tenue parce qu'il se sait observé par l'autre. L'interaction focalisée suppose qu'on accepte effectivement de maintenir ensemble et pour un moment un seul foyer d'attention visuelle et cognitive. Par exemple, une conversation, un jeu de table, une tâche conjointe occupent, dans un cercle étroit, des participants en position de face à face. Ceux qui maintiennent ensemble un foyer unique d'attention s'engagent certes également dans des interactions non focalisées. Mais ils ne le font pas en tant que participants d'une activité focalisée et les personnes présentes, étrangères à cette activité, participent aussi aux interactions non focalisées ${ }^{8}$. »

7 Les interactions non focalisées sont bien des interactions, avec toutes leurs conséquences normatives, même si la réciprocité des perspectives y est réduite à l'observabilité mutuelle. Ce sont des interactions dans la mesure où elles satisfont à une condition pour ainsi dire structurelle: elles mettent en rapport des dispositions sensorielles (la vue, l'audition, l'odorat, le toucher) et un langage corporel fait de mouvements, de gestes et d'attitudes. Ce rapport d'observabilité qui s'instaure dans la moindre coprésence est non seulement public mais publicisant: "Ordinairement, le fait de se servir de ses sens implique que l'on s'en serve à nu et que l'on se découvre par cet usage même ${ }^{9}$.» Dans la lignée de la «sociologie des sens» de Georg Simmel, on pourra ainsi rapporter les modalités de construction sociale de nos jugements à l'expérience de l'œil - le sens du jugement immédiat, celui par excellence de la métropole comme haut-lieu de la rencontre entre étrangers -, de l'oreille - le sens de la rumeur -, de l'odorat - le sens de l'intimité et de la répulsion ${ }^{10}$. On pourra aussi montrer comment un murmure ou un clin 
d'œil fonctionnent comme des «frontières focalisantes" qui limitent la sphère de propagation de l'information accessible.

8 Dans la mesure où les ressources constitutives des interactions non focalisées appartiennent à la sphère des informations prélevées au passage dans un champ de visibilité, elles relèvent de l'expérience routinière du passant. Le langage corporel qui se construit par la mobilisation de ces ressources et la lecture de ces indices est à la fois plus que la « conversation de gestes» de George Herbert Mead - parce qu'il suppose un sens partagé par l'acteur et le témoin et une forme minimale de réciprocité -- et moins qu'une symbolique universalisable - la signification des gestes ne va pas toujours de soi et implique un engagement spontané dans la situation en ce qu'elle a d'émergent. Les ressources de ce langage conventionnel et normatif sont donc disponibles localement et pour les participants, dans le jeu de leurs apparences concertées.

9 On conçoit que les compétences requises dans cet univers d'accessibilité réclament pour être analysées plus qu'un changement de focale de la part du chercheur. La métaphore optique ne saurait rendre compte ni des stratégies des acteurs dans cet univers, de leur sens des occasions et de leur opportunisme méthodique, ni du caractère local des conventions et des usages et donc de la diversité des cultures des lieux publics, ni surtout des formes de réciprocité à l'œuvre dans l'ordre des interactions. Une file d'attente ne se laisse décrire que dans les mouvements et le langage corporel de ceux qui la composent dans un lieu et pour un temps donnés. L'étude de son organisation et de sa dynamique demande donc que l'on s'intéresse au moment du rassemblement et que l'on adopte un situationnisme méthodologique à égale distance de l'individualisme méthodologique et du holisme confortable que la formule maussienne du phénomène social total, élevée aujourd'hui à la dignité d'un slogan, est censée résumer en guise de toute méthode. Les séquences d'activité et les situations de parole étudiées empiriquement ne livrent qu'un sens stratifié relevant de compétences qui ne s'intègrent que du haut d'une lecture symbolique. Placer la question de l'accord et de l'action conjointe en tête du programme de renouvellement des sciences sociales, c'est précisément se défier d'une pensée confortable de l'intégration des significations dans un même phénomène, montrer par exemple qu'il conjugue plusieurs échelles territoriales et plusieurs logiques d'usage, ou que la diversité des usages et des conventions brouille constamment les domaines de compétence respectifs des différents services et des corps professionnels qui les gèrent et impose aux opérateurs de coordonner des activités sur des registres inséparables mais distincts, de les traduire en passant d'un cadre et d'un espace mental à un autre. D'où la force de la figure du traducteur pour comprendre comment des connaissances, des perceptions différentes sont à la fois distribuées dans un espace de coopération en réseau et agencées dans les innovations d'un système technique.

La sociologie de la traduction et de l'innovation propose précisément de dépasser l'opposition entre contenus scientifico-techniques et contextes sociaux. Son concept central de réseau (réseau d'acteurs, réseau d'objets) vise à substituer une vision horizontale et « clinique » d'un milieu de travail ou d'un système d'activités quelconque, à son interprétation critique comme système de signes illustrant une logique de domination. Il y a loin pourtant entre l'ethnographie de laboratoire avec sa figure centrale de l'opérateur innovant, immergé dans les réseaux de son milieu technique, et la description des activités qui intéresse la sociologie urbaine. D'abord parce que le laboratoire des objets techniques en milieu urbain est ouvert à d'autres flux et que le rapport aux objets techniques est constamment « désolidarisé » ou publicisé, enchâssé 
dans un milieu plus large. Ensuite parce que les degrés d'expertise se distribuent de manière plus diffuse (professionnels, habitués, novices, occasionnels) sans une égale " solidarité technique ${ }^{11}$ » ne serait-ce que parce que l'opérateur est par ailleurs un usager comme les autres. Le fait qu'une connaissance ordinaire du système technique soit disponible des deux côtés du guichet institue un rapport particulier dans lequel la compétence technique est constamment convoquée au tribunal de la critique profane et privilégie la fonction de traduction du technique au profane. Cette condition est spécifique d'un domaine d'activité où les agents sont "en contact avec le public » et dont les produits sont des prestations, c'est-à-dire des liens de réciprocité actuels ou virtuels, présents ou différés. C'est dans ce domaine de la relation de service que les conflits de compétences techniques, contractuelles et civiles sont les plus explicites ${ }^{12}$. Exposées à la description indigène comme autant d'épreuves ordinaires de l'intelligence partagée, elles sont également, comme performances circonstanciées, accessibles à l'analyse comme des opérations dont il faut, à la suite d'un incident notamment, rendre compte. En somme, les compétences en question sont examinées deux fois: dans l'espace de justification du jugement ordinaire et par les «technologies de la justification ${ }^{13}$ » qui imposent aux systèmes techniques de restituer une description socialement acceptable de leur activité. Ajoutons enfin que, dans les métiers à forte solidarité technique du transport, et en particulier dans les métiers de la sécurité et de la régulation, cette double contrainte de publicisation, loin de porter à l'innovation, bloque les opérateurs sur des positions et des procédures confirmées, adaptées à la gestion des incidents et tendant à être routinisées ${ }^{14}$.

11 Si le sociologue de l'urbain entend donc contribuer à l'ethnographie des réseaux sociotechniques et des cultures de la traduction qui constituent leur milieu, il n'a aucune raison de privilégier les innovations sur les routines, ni de mépriser les acquis de l'éthologie sociale pour comprendre comment un réseau est mobilisé ou activé. Certes, les opérateurs dont il s'occupe ne sont pas que des primates mais la manière dont ils ne le sont pas relève d'une intelligence sociale que la primatologie étudie plus et en tout cas autrement que l'ethnographie de la vie de laboratoire.

\section{Les réciprocités du passage}

12 On sait depuis Ulf Hanner $z^{15}$ que la ville conjugue diversité (des cultures et des modèles culturels) et accessibilité. La porosité des espaces et le chevauchement des publics dans un espace de transport comme la Gare du Nord est d'abord un problème pour l'exploitant: comment penser ce pôle comme un monde, un ensemble de normes et de procédures cohérentes et générales susceptibles de s'appliquer aussi bien aux usages inter-urbains ou internationaux de la gare qu'à ses usages intra-urbains et locaux ? Quels types de prises et quelles intentions faut-il supposer à ces usages différenciés d'un seul et même espace de transition? Il désigne ensuite toute une série d'apories du métier d'architecte: que reste-t-il, dans une logique de l'accessibilité de la gare comme "bâtiment-voyageur»? Comment penser la forme physique et l'architecture sensible d'une « superposition de seuils »? Comment scénariser ou scénographier des usages aussi divers dans un espace qui se voulait cathédrale au XIx $x^{e}$ siècle et que les rituels ordinaires de la mobilité transforment en hangar à navettes? Parlant de la gare d'Osaka, un architecte dit ainsi que l'espace public y est défini "par une scénographie du parcours plus qu'à travers une forme architecturale construite", et que "cet espace non visuellement délimité, se perçoit à mesure qu'on le parcourt, séquence par séquence, 
selon un déroulement qui rappelle étrangement un spectacle télévisuel, ponctué d'effets, de métaphores, d'analogies. Il se découvre dans le temps du déplacement et non dans l'arrêt d'une contemplation ${ }^{16}$."

Ce monde qui échappe à la contemplation, monde imaginaire de l'insécurité ou du somnambule, monde pratique dans lequel on flotte et qu'organisent les routines de l'habitué, est un monde de passages. Cette forme, à la fois esthétique et civile, dans laquelle se conjuguent urbanité et modernité, a été largement explorée depuis Simmel et Benjamin. Elle est au cœur de l'inquiétude fascinée de Robert Park pour les processus de "désorganisation sociale ", c'est-à-dire d'hybridation culturelle, dont le migrant résume l'expérience.

14 On a suffisamment dit que la culture du passage est la culture urbaine par excellence et que la ville institue la pluralité des mondes et leur excentricité croissante. Culture de la diversité proche et de la distance radicale, qui fabrique de l'accessibilité et de la visibilité mutuelle sans échange. Mais peut-être n'a-t-on pas encore pris la mesure des conséquences théoriques, pour la recherche urbaine, de ce régime mutique du principe de réciprocité. Lévi-Strauss relu par Simmel. Les rituels de la place publique peuvent-ils être rangés sous les mêmes catégories que ceux de la table ? Lévi-Strauss, envoyé spécial dans les restaurants populaires du midi de la France, avait illustré à sa manière le principe kantien de l'insociable sociabilité : boire ensemble et manger seuls ${ }^{17}$. La grande métropole de Simmel nous filme dans un tramway et dans la réserve exposée des liens faibles: échanges de regards furtifs, plus question de boire ensemble, la visibilité mutuelle n'est pas la convivialité festive et le «nous », problématique.

Il fallait donc dresser un autre tableau des réciprocités, mettre la question de l'accord au ras du trottoir et observer les compétences du citadin dans la chorégraphie du passant. Or, à relire Goffman, à creuser la formule du passage jusqu'au " personnage conceptuel ${ }^{18}$ " du passant, la ville finit par bousculer les catégories de la pensée du social au rythme de "problèmes" dont la recherche ne maîtrisait ni les enjeux politiques (la mise entre parenthèses du civique au profit du civil), ni les conséquences théoriques (des pensées du système et de l'acteur aux pensées de l'action et de la situation). C'est dans cette mise aux normes de conjonction que les catégories subissent un grand écart. Compétences d'évitement ou d'arrangement avec leur régime d'interactions faibles ou flottantes. Compétences de participation et de coopération à toutes fins utiles. Mais s'agit-il d'une réduction? Certes, le passant est l'homme sans qualités qui passe d'un univers de compétences à l'autre: le monde subjectif dans lequel il évolue, l'expérience de la vie sociale qui est la sienne, témoignent de l'épuisement des pensées du « soi-même » et des ontologies du sujet. Mais ces pensées s'étaient avérées incapables de rejoindre le monde dont il s'agit, ce monde que nous ne contemplons pas. Parce qu'elles demeuraient organisées autour de deux métaphores de l'autrui qui ne conviennent pas à l'expérience urbaine. D'un côté, aboutissement d'une ontologie du sujet, le drame de l'apparition : la figure de l'insomniaque et le thème de l'insupportable et inaccessible altérité avec ses conséquences imaginaires - l'assomption, jusqu'à l'écœurement, de l'identité dans l'amour ou son implosion dans l'indifférence et l'évasion. De l'autre, le somnambule, être flottant et fragile, mobile et sans prise, dépendant d'autrui et de l'illusion de la familiarité qu'il lui assure. L'espace social du passant se construit précisément dans l'écart entre ces deux figures. Il n'est pas peuplé d'autruis et ses routines efficaces se déploient non dans l'espace de la familiarité, mais dans celui de l'étrangéité. L'hospitalité paradoxale et minimale que nous attendons d'un espace public urbain, espace de circulation susceptible 
d'être « visité » (Kant) par tout un chacun, et espace de rencontre avec l'étranger, veut qu'il soit accessible et nous offre des prises pour l'activité en cours, tout en ménageant la possibilité de se déprendre, d'évoluer dans un monde de liens faibles.

\section{Compétences et contextes}

16 On aura compris que la compétence majeure, dans cette culture urbaine de l'intervalle entre les mondes est une compétence de contextualisation. Celle-ci se définit par trois traits : l'aptitude à la commutation de code, la capacité à interpréter, dans un cours d'action ou de communication, des indices de contextualisation et à en tirer des conséquences, enfin l'aptitude à rendre compte de l'activité par une procédure de justification. Ce sont ces compétences dont nous sommes ordinairement acteurs et témoins que tente de décrire l'ethnographie des espaces publics.

17 Cette position de recherche s'appuie, depuis le projet énoncé par Dell Hymes et John Gumperz en 1964, sur une argumentation qui, loin d'être uniquement microsociologique est à la fois matérialiste ${ }^{19}$ et structurale. L'évolution des sociétés urbaines, dit Gumperz, met en contact non seulement des communautés homogènes, établies sur des territoires marqués sur le plan social et ethnique, mais des façons de parler, individuelles ou collectives, qui modifient le paysage culturel et les conditions dans lesquelles se fait le contact des langues entre elles. Ainsi, des « bribes de culture partagée » raniment ici ou là un sentiment commun d'appartenance en confortant la constitution de filières particularistes d'accès aux ressources, alors que, sur le plan linguistique, des marqueurs identitaires, s'exprimant de manière diffuse et pourtant efficace, nous rappellent que, dans notre vie quotidienne "nous dépendons de la coopération avec des gens qui ne partagent pas forcément notre culture ${ }^{20} »$.

18 Ces évolutions majeures des sociétés contemporaines vers une plus grande complexité et une plus grande dispersion culturelles (évolutions qui ne vont pas, faut-il le rappeler, sans réactions «purificatrices» de toutes sortes, territoriales et linguistiques), conduisent le sociologue et le linguiste à porter leur attention sur deux problèmes sociaux dont l'enjeu est à la fois scientifique et politique : d'une part, l'expérience des migrants vivant entre deux (ou plusieurs) cultures et l'usage qu'ils font de la pluralité des codes disponibles et, d'autre part, certaines situations-clés (entretiens d'embauche, interrogatoires, examens et concours administratifs, demandes d'allocations, etc.) où l'accès aux ressources est directement lié à l'évaluation à la performance communicative en situation. Ces deux problèmes mettent au premier plan de l'agenda des sciences sociales la question des formes concrètes de la négociation et de l'accord dans les situations de contact entre individus appartenant à des cultures différentes ou se référant à des registres de justification différents. Question qui fait appel à la fois aux notions de convention et de marché, les bousculant toutefois au profit d'une question politique, celle des compétences sociales nécessaires à la construction de normes communes.

Gumperz montre également que la commutation de code fonctionne comme une extension du lexique disponible, un enrichissement plutôt qu'un appauvrissement. Cette technique de communication comparable aux techniques de "plan rapproché " au cinéma, a l'intérêt d'évoquer pour les participants des éléments de contexte et des associations qui métaphorisent le propos et accentuent sa pertinence. Autrement dit, les façons de parler ne sont pas seulement à rapporter (à " corréler ») à des identités : les stratégies verbales sont pertinentes dans le contexte et l'enrichissent au cours de 
l'échange ${ }^{21}$. L'expérience du migrant entre deux mondes n'est donc que la métaphore de l'expérience du tout-venant, un terrain privilégié pour l'étude des processus par lesquels se construisent dans un contexte donné, les ajustements entre acteurs sociaux inégalement compétents ou inégalement disposés à se comprendre à demi-mot dans des situations problématiques.

Un contexte est donc bien plus que l'enveloppe d'une activité. C'est à la fois le cadre local et perceptif dans lequel se déroule une activité (setting), les éléments de l'environnement institutionnel et ethnographique qui servent d'arrière-plan à cette activité, et enfin l'espace de parole lui-même auquel les participants se réfèrent au cours d'un échange. La compétence d'un agent se définirait ainsi à trois niveaux, dans sa capacité à focaliser l'événement, à mobiliser les savoirs d'arrière-plan ou à interpréter le cours d'action. Tantôt il s'agit de repérer dans le contexte les indices permettant de hiérarchiser les niveaux d'attention, tantôt il s'agit de cadrer des attentes générales en s'appuyant sur l'expérience antérieure pour lever des ambiguïtés sur ce qui va suivre.

Mais dès lors que les contextes sur lesquels on travaille sont des événements de langage (par exemple, une annonce sonore ou la réponse à une réclamation), il est clair que le découpage doit porter sur la manière dont les « intentions communicatives » organisent le contexte. Autrement dit, la dynamique d'un contexte de communication, considéré comme événement de parole ou comme usage, est aussi une dynamique immanente susceptible d'être explorée de l'intérieur, dans le cours d'action, dynamique dont les ressources sont 1) produites par les locuteurs et les auditeurs, 2) disponibles pour chacun d'eux et 3) constamment évaluées par eux. Dans ce cours d'action, les participants s'emploient à parler «à propos » et tentent donc d'interpréter ce qui se dit et ce qui se passe.

Parler et agir en conséquence dans un contexte déterminé, c'est donc utiliser ses ressources immanentes, logées dans les plis de l'interaction et distribuées dans le cours de l'action ou de la conversation, qui appartiennent à des répertoires de rôles et de situations et les signalent pour ceux qui y sont impliqués. Les indices de contextualisation ont ainsi une double fonction : une fonction cognitive de cadrage et une fonction pratique et structurante. D'un côté, ils appartiennent à un contexte, ils sont formatés avec lui et par son contour - ce sont des ressources contextualisées; de l'autre, étant là pour être observés et interprétés, ils sont manipulés par les participants pour redéfinir et transformer le contexte - ce sont des dispositifs contextualisants. D'un côté, ce qui est dit donne lieu à des attentes sur ce qui va suivre et fonctionne comme une invite à la coopération; de l'autre, la manière de le dire, le moment où on le dit, attire plus ou moins l'attention de l'autre, ménage un changement de thème, ouvre un espace d'argumentation et témoigne d'une plus ou moins grande souplesse communicative.

Parce que nous sommes tenus d'être à la fois acteurs et observateurs des conséquences de nos actions, nous manipulons des cadres qui sont nos propres pièges, nous sommes des «faussaires captifs» (Goffman), jouant avec les interactions comme des criminels qui commettent leurs crimes en prison. Dans ce jeu, la capacité à rendre compte de nos activités, à lever un malentendu ou à préciser nos intentions, est une compétence dont on ne sait dire si elle est sociale ou morale, civique ou politique. Rendre compte c'est s'expliquer après coup d'un comportement fâcheux, d'un impair ou d'une gaffe et réduire le fossé entre une action et les attentes qu'elle vient de décevoir ${ }^{22}$. Dans les pratiques ordinaires de la conversation, les excuses et les justifications, tendent toutes deux à neutraliser un acte ou un propos, en l'isolant de ses conséquences, et à restaurer ainsi 
l'équilibre requis entre un personnage et son rôle. La fonction sociale et morale de ce type de rituel réparateur consiste à traiter les espérances déçues d'un public quelconque et à réduire l'écart entre une promesse ou une annonce et son accomplissement effectif. Les excuses et les justifications peuvent être considérées en ce sens comme des dispositifs élémentaires de l'évaluation des relations sociales. S'excuser c'est reconnaître son erreur ou sa faute mais, en ajoutant que l'on n'en est pas pleinement responsable, la mettre à distance de soi et l'évaluer : on invoque l'accident ou le lapsus, on plaide l'ignorance ou le manque de clairvoyance (On ne savait pas), l'impuissance du corps ou la toute-puissance des pulsions (On n'y pouvait rien, "C'était plus fort que moi»), on cherche un bouc émissaire (On s'est laissé entraîner par quelqu'un, on a subi son influence). Se justifier, en revanche, c'est accepter la responsabilité de l'acte que l'on vient de commettre tout en refusant l'évaluation péjorative qui en est faite. (On n'a rien fait de mal; les victimes méritent ce qui leur est arrivé ; d'autres font bien pire sans qu'on les poursuive; il fallait bien venger une injustice antérieure.) Il y a également «les tristes histoires» (les sad tales) qui rendent compte de la situation en la replaçant dans un récit ou dans une trajectoire typique de victime ou de perdant. Toutes ces techniques par lesquelles nous "racontons des histoires aux situations » lorsqu'elles tournent mal, relèvent d'un vocabulaire de la réparation, dont nous disposons dès les premières étapes de la socialisation. La compétence sociale que traduit ce vocabulaire est partie intégrante de notre formation puisqu'elle nous permet de continuer à parler et de nous présenter en public, alors même que les distorsions ou les contradictions de nos rôles se manifestent au grand jour.

En résumé, contextualiser c'est prendre soin de la pertinence d'un cours d'action et en modifier éventuellement l'intelligibilité pour ceux qui y sont impliqués; rendre compte, c'est accorder au public ou à l'auditoire un pouvoir de juger avec lequel on ne peut négocier que si l'on en accepte les principes et si l'on admet l'existence d'un «tribunal de la critique profane », celui de la raison commune.

Parce qu'un rassemblement n'est pas un groupe dont on analyserait la sociabilité dans les formes du "nous ", les différentes façons de dire l'accord et d'afficher sa compétence doivent être « redonnées », reprises comme de nouveaux observables. Il s'agit là d'un pari et d'une croyance en un monde exposé, qui n'est monde que dans cette exposition-même, dans la rue ou la conversation. Cette conversion empiriste n'a que peu à voir avec le cheminement initiatique d'un chercheur présumé «idiot culturel», inapte au changement de milieu, encombré de préjugés qui ne seraient solubles que par immersion. De ce point de vue, les espaces publics du transport collectif nous ont beaucoup aidé : les « indigènes » qui l'exploitent loin d'être reclus dans un autre monde sont en contact constant avec le public, et le milieu d'activités qu'il s'agit d'étudier se définit précisément comme un lieu de passage et non de résidence et encore moins d'immersion. Il faut donc proposer de l'empirisme et de la conversion empiriste une définition plus générale et moins normative. Non pas conversion comme contrainte, mais comme intention et comme implication: l'empirisme n'est pas le noyau dur et le dernier recours de la sociologie critique en crise, mais l'expérience de la crise du système des positions constitutif de ce qu'on appelle un terrain, et qui en règle l'accès et la possibilité de l'interpréter. La conversion empiriste est une croyance avant d'être une méthode ${ }^{23}$.

De quelle manière la ville nous invite-t-elle à croire une nouvelle fois et d'une autre manière au monde? C'est sur ce terrain que la sociologie urbaine peut tenter de contribuer, pour sa part, au traitement de la question politique des compétences du 
citadin. Et elle ne peut le faire qu'en parasitant les vocabulaires traditionnels de l'engagement - participation, mobilisation, assentiment -, en affranchissant la pensée de l'action dans l'espace public de ses métaphores rationnelles pour l'impliquer dans les procédures et les disputes concrètes du parler ordinaire. La conversion empiriste ne peut donc se concevoir qu'au rebours de la pensée critique, comme conversion à l'apparence ${ }^{24}$. Empirisme donc, non comme respect mécanique des données mais redécouverte de l'existant, de ce qui se rend visible, de ce qui se présente comme être-en-commun, en même temps que de l'intervalle spatio-temporel dans lequel la pluralité se présente. L'accord que vise le chercheur avec ce qu'il observe est tout à la fois politique et pratique : il ne se donne pas un idéal de familiarité et ce n'est pas un état (intégration, immersion), mais un désir et une croyance, un mouvement du "deux-en-un » de la pensée intègre ${ }^{25}$, de la pensée soucieuse de son impuissance, incapable de monter en généralité en mobilisant les vocabulaires disponibles. Ce sont là des troubles assez communs de l'observation pour qu'on les prenne au sérieux à partir de l'espace de la ville qui a constitué pour eux un terrain favorable. Ce ne sont plus les troubles répertoriés du face à face avec l'indigène ou du changement du milieu, mais ceux qui tiennent à la définition de notre part d'indigénéité ou aux difficultés que nous avons à penser ensemble la porosité et l'étrangéité des milieux.

\section{NOTES}

1. Cf. G. Simmel, « La mode », in La tragédie de la culture, Paris, Rivages, 1988, p. 101.

2. Cf. I. Joseph, D. Boullier, V. Guillaudeux, E. Lévy, M. Lacoste, D. Bayart et A. Borzeix, Gare du Nord: mode d'emploi, Paris, Éd. Recherches, Plan urbain/RATP-SNCF, 1995 ; I. Joseph, « Ariane et l'opportunisme méthodique », Annales de la Recherche urbaine, 71, Gares en mouvements, 1996, p. 5-13.

3. B. Lepetit, "Histoire des pratiques, pratiques de l'Histoire", Introduction à Les formes de l'expérience. Une autre histoire sociale, Paris, Albin Michel, 1995, p. 9-22.

4. Cf. E. Goffman, La mise en scène de la vie quotidienne, t. 2, Paris, Minuit, 1973, p. 21-33.

5. Par compétence, B. Lepetit entend «la capacité à reconnaître la pluralité des champs normatifs et à identifier leurs contenus respectifs ; l'aptitude à repérer les caractéristiques d'une situation et les qualités de ses protagonistes; la faculté, enfin, de se glisser dans les espaces interstitiels que les univers de règles ménagent entre eux, à mobiliser à leur profit le système des normes et des taxinomies le plus adéquat, à construire, à partir de règles et de valeurs disparates, les interprétations qui organiseront différemment le monde. Sur tous ces points, aucune égalité n'est postulée entre les acteurs. Leur liberté est en proportion de leur position du moment, de la multiplicité des mondes auxquels leurs expériences biographiques leur ont donné accès, et de leurs capacités inférentielles », B. Lepetit, " Histoire des pratiques, pratiques de l'Histoire », op. cit., p. 20.

6. U. Hannerz (Explorer la ville. Éléments d'anthropologie urbaine [1980], Paris, Minuit, 1983) avait proposé de distinguer anthropologie dans la ville et anthropologie de la ville. L'anthropologie dans la ville définit son objet comme un milieu localisé (le quartier chinois de New York ou Paris, le milieu de la délinquance...) où le chercheur tente de se faire une place pour en comprendre 
l'organisation interne et le mode de vie. Encore faut-il ne pas mythifier l'histoire de cette position de recherche. Contrainte avec la décolonisation de «rapatrier" son exotisme, l'anthropologie urbaine a dû prendre en compte au moins deux faits nouveaux : la participation de l'indigène à la définition de son observabilité et la diversité des cadres de participation dans lesquels s'engage le chercheur avec le milieu qu'il observe. Quant à l'anthropologie de la ville, notons que, à l'opposé d'un quartier populaire ou d'une cité de banlieue, une gare ou une station de métro n'est pas un milieu dans lequel on s'établit. La singularité « urbaine » du rapport du chercheur à son objet le situe d'emblée dans une pluralité de champs normatifs entre lesquels il va et vient. Le retour au terrain mobilise alors des compétences (de traducteur ou d'informateur) également mobilisées par d'autres procédures d'enquête, qui ne sont pas celles de l'immersion dans un monde étrange ou lointain, mais de l'accord entre plusieurs mondes proches et ordinaires.

7. J.-C.Chamboredon et M. Lemaire, «Proximité spatiale et distance sociale. Les grands ensembles et leur peuplement », Revue française de Sociologie, XI, 1970, p. 3-33.

8. E. Goffman, Encounters, Indianapolis, Bobbs-Merrill, 1961, p. 7.

9. E. Goffman, Behavior in Public Places, Glencoe, The Free Press, 1963, p. 16.

10. G. Simmel, "Essai sur la sociologie des sens", in Sociologie et épistémologie, Paris, Presses universitaires de France, 1981, p. 223-238.

11. N. Dodier, Les hommes et les machines, Paris, Métailié, 1995, p. 7-95.

12. I. Joseph et G. Jeannot, eds, Métiers du public, Paris, Éd. du CNRS, 1995.

13. L. Suchman, «Constituting Shared Workspaces », Xerox Palo Alto Research Center, 1991.

14. D. Boullier, « Histoires urgentes », in Gare du Nord : mode d'emploi, op. cit., p. 27-136.

15. U. Hannerz, Explorer la ville..., op. cit.

16. T. Roty, «EKI, le relais : la gare au Japon », Annales de la Recherche urbaine, 71, 1996, p. 75-85.

17. M. Hénaff, Claude Lévi-Strauss, Paris, Belfond, 1991, p. 48.

18. G. Deleuze et F. Guattari, Qu'est-ce que la philosophie ?, Paris, Minuit, 1991.

19. Voir D. Hymes : «Un enfant acquiert une connaissance des phrases, non seulement comme grammaticales, mais aussi comme étant ou non appropriées. Il acquiert une compétence qui lui indique quand parler, quand ne pas parler, et aussi de quoi parler, avec qui, à quel moment, où, de quelle manière. "Vers la compétence communicative, Paris, Hatier, 1981, p. 74. Hymes, en pastichant les thèses sur Feuerbach de K. Marx, se démarque de Chomsky en ces termes : «La linguistique cartésienne fait de l'essence linguistique, l'essence de l'homme. Mais l'essence de l'homme n'est pas une abstraction inhérente à chaque individu. Dans son actualité réelle, c'est l'ensemble des rapports sociaux. La linguistique cartésienne qui ne va pas jusqu'à la critique de cette essence actuelle est donc conduite : 1) à procéder à des abstractions du procès historique, à établir une intuition linguistique comme quelque chose qui se réfère à soi-même et à présupposer un individu humain abstrait et isolé ; 2) à considérer l'essence de l'homme comme espèce, c'est-à-dire comme cette généralité plate qui unit les individus dans leur naturalité, et non socialement. " «La linguistique ne suffira pas tant qu'elle ne prendra pas en compte les manières de parler dans leur rapport aux situations et aux significations sociales, tant qu'elle n'admettra pas que le point de départ de la description n'est pas un énoncé ou un texte mais un événement de langage, non une langue mais un répertoire des manières de dire, non une communauté de langage définie par correspondance avec une langue mais une communauté de langage définie par le rapport conflictuel entre des règles de grammaire et des règles d'usage. » Foundations in Sociolinguistics, Philadelphie, University of Pensylvania Press, 1974. Sur les notions de « code-switching » et d'indices de contextualisation, voir J. Gumperz, Engager la conversation, Paris, Minuit, 1989.

20. Ibid., p. 8.

21. Les "situations-clés", dont l'enjeu pour les locuteurs est plus direct, sont celles qui les confrontent à une évaluation de leur performance et décident de leur emploi, de leur carrière ou 
de leur statut, en jugeant de leurs capacités à mobiliser leurs ressources communicatives en situation. Gumperz étudie ainsi des entretiens d'embauche en soulignant que la maîtrise de la langue par des locuteurs étrangers ne suffit pas à aplanir les préjugés ou les discriminations. L'intonation, l'accent, le style argumentatif sont autant d'indices dont se saisissent ceux qui participent à un événement de langage pour cadrer et comprendre le contexte et sa signification, suppléer parfois à l'absence d'une terminologie spécifique ou d'une nomenclature rigide et gérer des accords nécessairement partiels et vulnérables.

22. M. B. Scott et S. M. Lyman, " Accounts ", American Sociological Review, XXXIII, 1968, p. 46-62.

23. «Il se peut que croire au monde, en cette vie, soit devenu notre tâche la plus difficile, ou la tâche d'un mode d'existence à découvrir sur notre plan d'immanence aujourd'hui. C'est la conversion empiriste (nous avons tant de raisons de ne pas croire au monde des hommes, nous avons perdu le monde, pire qu'une fiancée, un fils ou un dieu...). Oui, le problème a changé. » G. Deleuze et F. Guattari, op. cit., p. 72-73.

24. Citons E. Tassin commentant Hannah Arendt : «Il faut aborder l'activité de penser à partir de la vie pour en déceler la condition politique et rendre non pas la cité digne de la pensée, mais la pensée digne de la cité. Cette condition, qui est aussi la condition humaine du monde, et au monde, peut être mise au jour dans la question de l'apparence. » "La question de l'apparence », in Colloque Hannah Arendt. Politique et pensée, Paris, Payot, 1996, p. 67-89.

25. E. Tassin, ibid.

\section{RÉSUMÉS}

Quelles sont les propriétés du rassemblement et les ressources régulatrices de l'accessibilité ? Nulle part mieux que dans une gare ne se vérifie l'idée selon laquelle un espace public est un agencement de domaines contestés ou contestables. L'accessibilité ne peut s'y réduire à la capacité d'un lieu à interagir avec d'autres lieux. Cette première définition, géographique et fonctionnelle, renvoie à la ville comme espace de circulation et de déplacements. Mais l'accessibilité est également la qualité d'intelligence partagée d'un espace de communication et désigne alors les qualités d'une prestation à ne pas être simplement un "message ", d'un équipement à être plus qu'un instrument, et les ressources qu'ils offrent tous deux pour confirmer ou réguler les dimensions dialogiques de l'espace d'activité. À partir de la gare, il s'agit donc de comprendre comment la ville peut modifier notre perception du social et de l'histoire sociale, d'analyser la construction de l'urbanité dans ses catégories propres et de retrouver la trace de l'expérience urbaine dans les enquêtes sur les compétences sociales des citadins.

What are the properties of gathering and regulatory resources of accessibility? The idea that a public space is an agency of contested or contestable domains is nowhere better verified than in a railway station. Accessibility cannot be reduced to the capacity of a place to interact with other places. This initial geographical and functional definition reveals the city as a space of circulation and movement. But accessibility is also the quality of shared intelligence of a place of communication. It then indicates qualities of a service more than a simple "message", of a future equipment and the resources offered by both to confirm or regulate the dialogical dimensions of the space of activity. The point in question is thus to understand, from the basis of the railway station, how the city can modify our conception of the social and of social history. It is to analyse 
the construction of urbanity in its own categories and rediscover the trace of the urban experience in the inquiries into the social skills of town dwellers.

\section{AUTEUR}

\section{ISAAC JOSEPH}

Isaac Joseph (Université de Paris X-Nanterre) a publié avec Y. Grafmeyer, L'Ecole de Chicago : Naissance de l'écologie urbaine, Paris, Aubier, $3^{\mathrm{e}}$ éd., 1991 ; Le passant considérable : Essai sur la dispersion de l'espace public, Paris, Méridien, 1984. Il a également dirigé plusieurs ouvrages collectifs : Le franc-parler d'Erving Goffman, Paris, Editions de Minuit, 1989 ; Prendre place : Espace public et culture dramatique, Paris, Editions Recherches-Plan urbain ; avec G. Jeannot, Métiers du public : les compétences de l'agent et l'espace de l'usager, Paris, Editions du CNRS, 1995 ; avec J. Proust, La folie dans la place, Paris, Editions de l'EHESS, 1996. 